\title{
Der weite Blick: Wirkung und Reichweite umsichtiger Planung. Versuch, eine exemplarische Geschichte kurz zu berichten am Beispiel der Zentral- und Hochschulbibliothek Luzern und der Kooperativen Speicherbibliothek Schweiz
}

\begin{abstract}
Die Planung von Bauprojekten ist oft nicht nur komplex, sondern dauert auch längere Zeit - eine Erfahrung, die die ZB Zürich und die ZHB Luzern teilen. Die Notwendigkeit, die Zentral- und Hochschulbibliothek Luzern zu erweitern und neuen Bedürfnissen anzupassen, führte $\mathrm{zu}$ einer umfangreichen Serie von Raumplanungs-, Umnutzungs- und Machbarkeitsstudien ohne Realisierungslösung. Erst als die Resultate einer Detailstudie dazu zwangen, den Blick weit über die eigene Institution hinaus zu heben, zeichnete sich die Lösung klar und einfach ab. Der folgende Aufsatz beschreibt die Erweiterung der ZHB Luzern, den Aufbau der Kooperativen Speicherbibliothek Schweiz und die Renovation des Haupthauses der ZHB Luzern im Spannungsfeld von weitsichtiger Sachplanung und Primat der Politik.
\end{abstract}

\section{Einleitung}

In der Kochkunst lässt man gerne das Fleisch am Knochen einige Zeit ruhen, ,abhangen‘. Bei Projekten scheint es ähnliche Phänomene zu geben, vor allem bei Bauprojekten: Hier können die ZB Zürich und die ZHB Luzern, bei allen sonstigen Unterschieden, mehrere längere Lieder gemeinsam davon singen, wie sich solches Abhangen hinziehen und gelegentlich über alle kochkünstlerische Vernunft hinaus andauern kann. Ob eine Veranstaltung mit diesen Liedern allerdings viel Publikum anzöge?

\footnotetext{
Anmerkung: Direktor der ZHB von 1995 bis 2017. Die Bauprojekte der Bibliothek und die Vertretung der Bibliothek in den Baugremien gehörten zu seinen wichtigsten und gewichtigsten Dossiers. - Auch neben seiner eigenen Bibliothek beschäftigten ihn Fragen des Bibliotheksbaus intensiv, als Mitglied der LIBER Architecture Group von 1996 bis 2018 und als deren Chairman von 2006 bis 2016.
}

๑ Open Access. ๑ Ulrich Niederer, publiziert von De Gruyter. $\Subset$ BY-NC-ND Dieses Werk ist lizenziert unter der Creative Commons Attribution-NonCommercial-NoDerivatives 4.0 Lizenz. 
Ich riskiere trotzdem, in der Folge die Prosaversion eines solchen Liedes zu erzählen. Es hätte eigentlich viele Strophen, aber ähnlich wie bei Kochsendungen werde ich mich auf die wichtigen Schritte konzentrieren - das ganze Lied wäre nicht nur eine lange Weile zu lang, sondern ich könnte in den Verdacht geraten, im Wettbewerb mit der ZB stehen zu wollen. Denn das grosse Erweiterungsprojekt der ZB dauerte ja bekanntlich rund 36 Jahre (so wurde an der Eröffnung 1994 deklariert), während die ZHB in diesem Jahr ins stolze 38. Planungsjahr eingetreten ist - mit ein wenig Glück (das vor allem braucht es dieser Tage in Luzern) wird sie ihre Eröffnung des sanierten und zu neuer Nutzung ertüchtigten Hauptgebäudes zusammen mit dem 40-Jahr-Jubiläum der Aufnahme der Planung begehen können. Das Projekt hat immerhin schon fünf Regierungsräte erlebt, drei Kantonsbaumeister beschäftigt und es arbeitet am vierten Bibliotheksdirektor...

Aber es gibt einen weiteren guten Grund, von Luzerner Wegen und Umwegen und schliesslich, ja, von Erfolgen in der Festschrift für Susanna Bliggenstorfer und zu ihrer Zeit als Direktorin der ZB Zürich zu erzählen. Denn da, wo das Projekt Erfolge vorzuweisen hat, sind sie umsichtiger Planung und dem weiten Blick zu verdanken. Umsichtige Planung und weiter Blick über die eigene Einrichtung hinaus: Das hat Susanna Bliggenstorfer immer wieder eingefordert in den grossen, oft nationalen Projekten, in denen sie sich engagierte, nicht selten leitend, und sie hat es im eigenen Haus selbst praktiziert, für die physische wie für die konzeptionelle Entwicklung der ZB Zürich.

\section{Die Zentral- und Hochschulbibliothek Luzern (ZHB)}

1951 wurde das neue Gebäude der Zentralbibliothek (ZB), wie sie damals noch hiess, ${ }^{1}$ eröffnet, unter Beifall der schweizerischen und der internationalen Bibliothekswelt. Sie bot ca. 75 Arbeits- und Leseplätze für Benutzende, einen unabhängig vom normalen Bibliotheksbetrieb zugänglichen Vortragssaal sowie Büros für etwa 15 Mitarbeitende, schliesslich einen separaten Bürobereich für einen anderen Teil der kantonalen Verwaltung mit rund 20 Büroarbeitsplätzen. Das Magazin war ausgelegt für rund 400'000 Bände; beim Einzug brachte die Bibliothek 260’000 Bände mit, hatte also reichlich Zuwachsplatz. Die Lage ist exzellent: zwei Minuten vom Bahnhof entfernt, in einem kleinen Stadtpark gelegen.

1 „Zentralbibliothek“ (ZB) war der offizielle Name von 1951 bis 1999, seit 1999 heisst die Institution „Zentral- und Hochschulbibliothek“ (ZHB). 


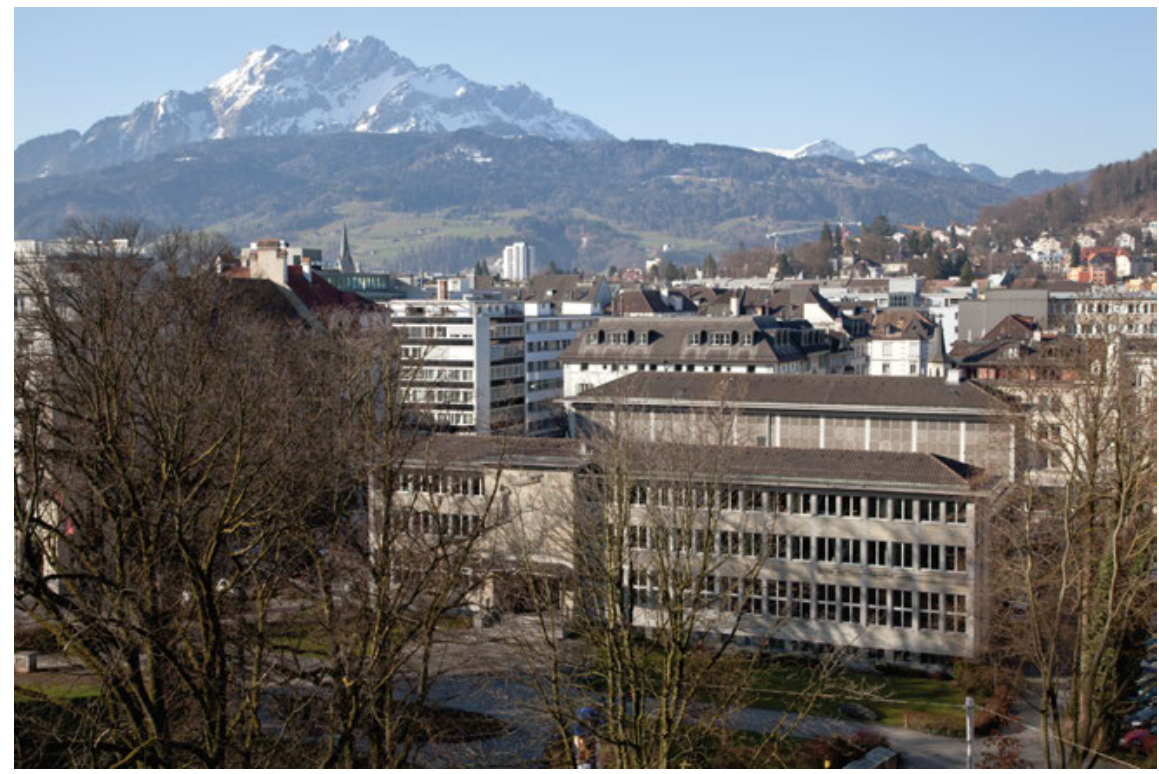

Abb. 1: ZHB im Sempacherpark (@ Ulrich Niederer, ZHB Luzern)

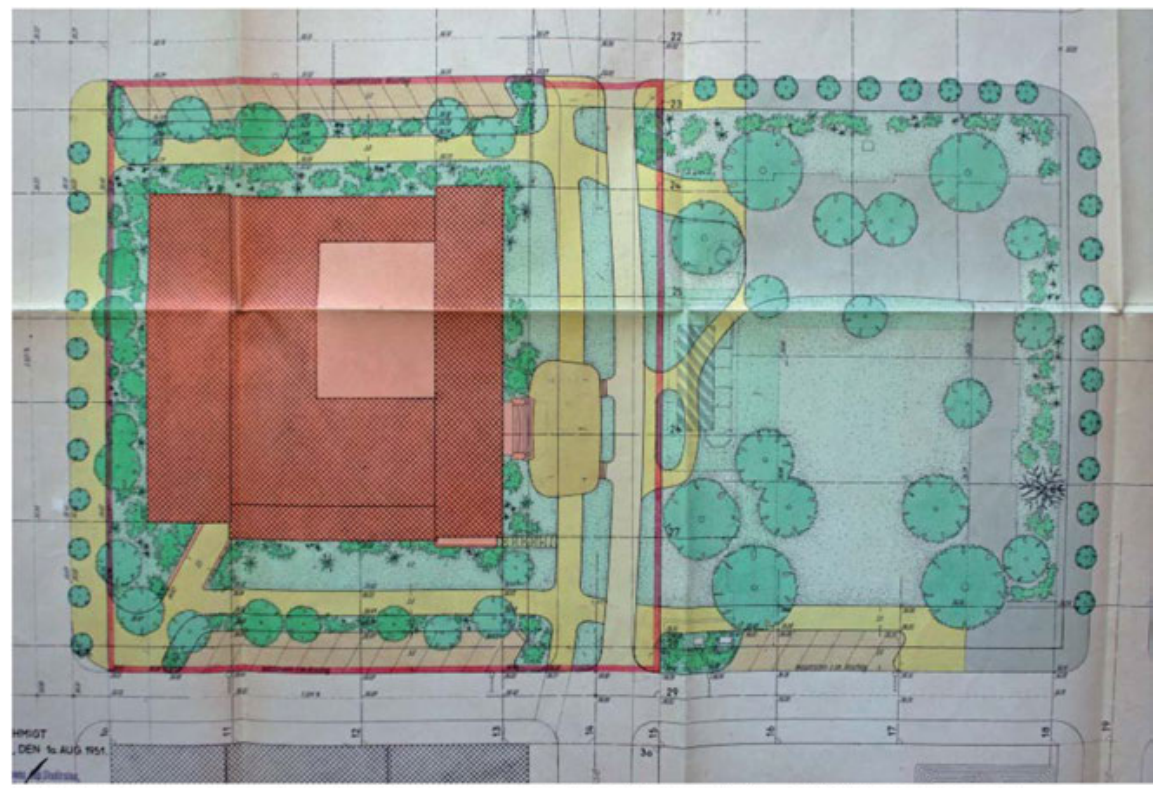

Projekt Umgebung und Verkehrsführung, Vom Stadtrat Luzern genehmigtes Projekt vom 10. August 1951 (Staatsarchiv Luzern)

Abb. 2: Situationsplan von 1951: die Bibliothek mit Innenhof im Sempacherpark (๔ Otto Dreyer, Staatsarchiv Luzern) 
Weniger als 30 Jahre später begann die Bibliothek aus allen Nähten zu platzen. Sie wies einen Bestand von über 500`000 Bänden auf und hatte über 30 Mitarbeitende. In den Magazinen begann man Notgestelle aufzustellen und im Soussol wurden Rollregalanlagen eingebaut und Büros für die Mitarbeitenden eingerichtet. Der Regierungsrat setzte eine Arbeitsgruppe ein, die Lösungen für den Platzmangel erarbeiten sollte.

Wieder 30 Jahre später (die ZHB ist mittlerweile auch Bibliothek für die Universität Luzern, für Teile der Fachhochschule Zentralschweiz [der Hochschule Luzern] und für die Pädagogische Hochschule Luzern, hat über 1 Mio. Bände und über 100 Mitarbeitende), nach zahllosen Arbeitsgruppen, Studien und Plänen und drei Bibliotheksdirektoren -, hat das kantonale Parlament auf der Basis eines Entwicklungsplans für die Luzerner Hochschullandschaft entschieden, die ZHB umzubauen und zu renovieren.

\section{Bildung im Kanton Luzern}

Aber vorerst ist es notwendig, einen kurzen Blick auf die Entwicklung der Hochschul-Bildung im Kanton Luzern und in der Stadt Luzern, dem natürlichen Zentrum der Zentralschweiz, zu werfen.

Luzern hatte seit dem 17. Jahrhundert eine Universitäre Hochschule, die zuerst eine katholische Theologische Fakultät, später auch eine Geisteswissenschaftliche Fakultät umfasste. 1978 lehnte der Souverän ein grosses Aufbauprojekt für eine Volluniversität ab. Aber im Verlauf der 80er Jahre kam es zu einem Ausbau vor allem der Höheren Fachschulen, der Vorläufer der Fachhochschulen. 1994 legte die Regierung des Kantons einen Planungsbericht zur Bildung im Hochschulbereich vor, in dem sie ihr Konzept zum Aufbau der Fachhochschulen und zum Ausbau der universitären Strukturen zu einer kleinen Universität Luzern darlegte. Das Parlament stimmte dem Bericht zu; damit war die Bahn frei für eine stürmische Entwicklung der Hochschullandschaft in der Region Zentralschweiz (Kantone Luzern, Zug, Nidwalden, Obwalden, Schwyz, Uri).

Das Konzept beinhaltete auch, dass die Zentral- und Hochschulbibliothek für die Institutionen des Hochschulbereichs die bibliothekarische Versorgung übernimmt. Die Zentral- und Hochschulbibliothek hat damit (wie ja die meisten grossen akademischen Bibliotheken in der Schweiz) eine doppelte Funktion: einerseits als Bibliothek die Forschung und Lehre der Universität, der Hochschule Luzern und der Pädagogischen Hochschule Luzern zu unterstützen und andererseits dem allgemeinen Publikum den Zugang zu nicht nur akademischer Literatur zu gewährleisten. Hinzu kommt die Aufgabe des Sammel- und Aufbewahrungszentrums für das regionale Dokumentenerbe, von mittelalterlichen Manuskripten 
und wertvollen Drucken über Nachlässe von Personen, Institutionen und Firmen bis zu Kleinschriften und audiovisuellen und elektronischen Publikationen.

Der markanteste Schritt in der Hochschulentwicklung des Kantons war zweifellos der positive Volksentscheid zum ,kleinen` Ausbau der Universität Luzern im Jahr 2000. Er hat für die Bibliothek in den letzten 17 Jahren zu einer Vermehrung des Personalbestandes um 50\% und des Buchbestandes um gleich viel geführt! Diese Entwicklungen haben aber die räumlichen Probleme der Bibliothek rasch verschärft. Denn einerseits hat mit der Zunahme der Bestände auch der dringende Bedarf an Platz zur Aufbewahrung derselben zugenommen, umso mehr, als die Universität mit ihren neuen und alten Instituten zunächst in kleinräumigen Provisorien untergebracht wurde in Erwartung des neuen Gebäudes, das im Herbst 2011 eröffnet wurde. Die geisteswissenschaftliche Ausrichtung der Universität mit ihren vier Fakultäten Theologie, Kultur- und Sozialwissenschaften, Recht und, seit 2016, Wirtschaft erlaubt es auch nicht, einfach nur auf elektronische Informationsträger zu setzen - das sind zumeist Fächer, die noch immer stark auf gedruckte Materialien setzen. Und andererseits wurden alle grösseren Renovationsarbeiten am Hauptgebäude der ZHB zurückgestellt in der Erwartung der Realisierung des umfassenden Konzeptes zur Erweiterung und Sanierung. Das führte dazu, dass der Zustand des Hauses in manchen Teilen prekär und die Platzverhältnisse vielerorts dysfunktional eng wurden.

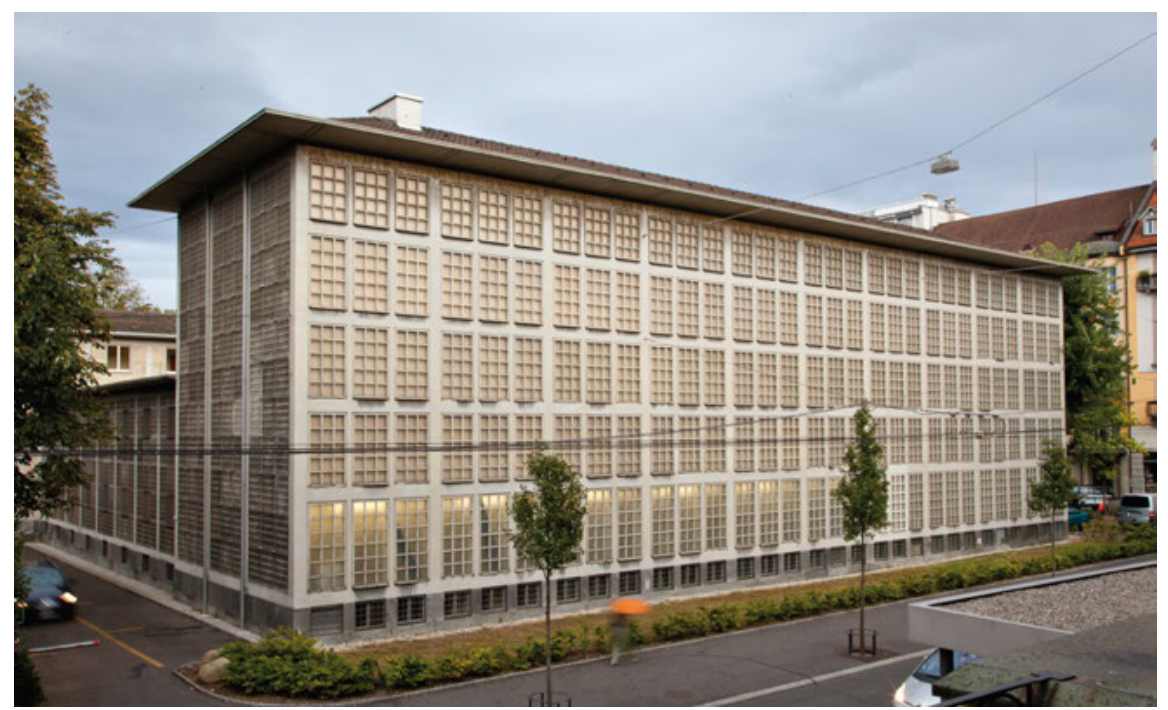

Abb. 3: ZHB, Magazin an der Hirschmattstrasse. Die teils schadhaften, abgebrochenen Simse sind deutlich sichtbar. (○ Ulrich Niederer, ZHB Luzern) 
Diese Situation des Raummangels wurde nicht einfacher durch den Entscheid der kantonalen Gebäudeversicherung, im sechsstöckigen Magazinbereich die obersten drei Geschosse wegen fehlender Fluchtwege für die interne Nutzung zu sperren.

\section{Klärungen auf dem Weg zur neuen Bibliothek}

Nach dem Entscheid, die Universität Luzern auszubauen, folgte sofort die Planung eines Gebäudes. Gleichzeitig wurde auch die Planung für die Räumlichkeiten der ZHB wieder aufgenommen; die kantonale Baubehörde gab insbesondere zwei Machbarkeitsstudien in Auftrag, die zu zentralen Erkenntnissen für diese Planung führten.

Die erste von 2001 untersuchte die Möglichkeiten und Grobkosten der Erweiterung der Bibliothek am bestehenden Ort und verglich sie mit einem kompletten Neubau. Diese Machbarkeitsstudie ergab drei wichtige Resultate:

1. Das Raumprogramm, das die Bedürfnisse der Bibliothek festhielt und quantifizierte, wird bestätigt. Es enthält vor allem einen grosszügigen Freihandbereich (bis dahin war die ZHB eine reine Magazinbibliothek!), eine grössere Zahl an Arbeitsplätzen für Benutzende und für Mitarbeitende, eine markante Verbesserung der Benutzungs- und Ausstellungsmöglichkeit für die Sondersammlung sowie eine Klärung des Layouts für die Betriebsabläufe.

2. Eine Erweiterung am bestehenden Standort ist nicht mit sinnvollen Mitteln möglich; zu sehr schränken sowohl das vorhandene Gebäude als auch die Umgebung - der Park, der mit seinem alten Baumbestand nicht in die Planung einbezogen werden durfte - die Möglichkeiten ein.

3. Ein vollständiger Neubau würde, ob am selben oder an einem anderen Ort, nicht unter CHF 40 Mio. kosten. Diese Summe, das machte der Regierungsrat, die Exekutive des Kantons, unmissverständlich klar, kann für die Bibliothek auch langfristig unter keinen Umständen zur Verfügung gestellt werden; möglich sind maximal CHF 20 Mio. Daraus ergab sich ein prinzipieller Entscheid: Das bestehende Gebäude kann allenfalls renoviert, eine Erweiterung muss an einem zweiten Standort realisiert werden, idealerweise im neuen Gebäude der Universität, in dem die aktuellen Bestände für den Lehr- und Forschungsbetrieb ihren adäquaten Standort hätten.

An diese Resultate anschliessend untersuchte die zweite Machbarkeitsstudie von 2004/2005 die Möglichkeiten und Kosten einer Renovation vor allem des Magazintraktes. Dieser Teil des Gebäudes weist zwei Hauptschwierigkeiten auf: Zum einen ist er in einem schlechten baulichen Zustand, zum anderen stellen die 
Büchergestelle die statisch tragende Struktur dar. Das ist nicht nur ausgesprochen unflexibel, sondern auch sehr gefährlich in einem Brandfall. Wenn in einem unteren Geschoss ein Feuer ausbricht, so werden innerhalb kurzer Zeit Temperaturen erreicht, die den Stahl der Büchergestelle schmelzen lassen - ein Angriff auf den Kern der Statik! Eine Sprinkleranlage ist nicht eingebaut; sie könnte auch nur mit dramatischen Verlusten an Stellplatz für die Bestände realisiert werden.

Diese Machbarkeitsstudie kam zum Schluss, dass eine reine Renovation zwar möglich ist, aber das Kosten-Nutzen-Verhältnis ganz ungünstig ausfällt, nicht zuletzt deswegen, weil der dringend benötigte Ausbau des Magazins auf eine Kapazität von mindestens 1,5 Millionen Bänden an diesem Standort nicht möglich ist. Die ZHB steht in einem Gebiet mit einer ausgedehnten Grundwasser-Schicht. Frühere Tiefbauversuche bei anderen Bauten verursachten gefährliche Risse in zahlreichen Gebäuden des Quartiers. Seither sind tiefe Bauten in diesem Gebiet per Gesetz verboten. Eine Umnutzung des Magazintraktes in einen Freihandbereich für das Publikum ist möglich; allerdings muss die statische Grundstruktur verändert werden. Ein Neubau schliesslich des Magazintraktes ist ebenfalls möglich, aber wiederum mit einem schlechten Kosten-Nutzen-Verhältnis verbunden. Zudem bringt ein Neubau nur des Magazintraktes keine betrieblichen Vorteile.

Die Arbeitsgruppe, die die zweite Machbarkeitsstudie begleitete - sie umfasste Vertreter verschiedener Behörden aus Stadt und Kanton, darunter auch die Denkmalpflege, den Stadtarchitekten und den Kantonsbaumeister sowie den Direktor der Bibliothek -, fällte auf der Basis dieser Resultate prinzipielle Entscheide, die Grundlage für die weitere Planung wurden:

1. Der Magazintrakt muss dringend renoviert werden,

2. am sinnvollsten ist eine Umnutzung dieses Gebäudeteils und

3. das Gebäude ist architektonisch wertvoll, so dass es als Ensemble erhalten werden soll. Auch die Höhe des Gebäudes kann nicht ohne aufwendige Umzonung verändert werden; aus städtebaulicher Sicht ist zudem ein höheres Gebäude in diesem Park und in diesem Stadtraum unerwünscht.

\section{Konzept}

Zeitgleich mit dieser Machbarkeitsstudie wurde auch definitiv festgelegt, an welchem Standort das neue Gebäude der Uni entstehen soll: unmittelbar hinter dem Bahnhof, in einem Betriebsgebäude der Post. Das Gebäude wurde zwischen November 2007 und April 2011 umgebaut und am 01. September 2011 eröffnet. Es umfasst mehr als 22’000 m² und enthält die Räume für den gesamten Unterrichtsbetrieb der Universität Luzern und für einen grossen Teil der PH Luzern, dazu auf rund 5’000 m² den Teil der ZHB, der diese beiden Institutionen unmittelbar unter- 
stützt. Die Universität und die PH Luzern hatten damals zusammen rund 4'000 Studierende.

Die beiden Machbarkeitsstudien für die Bibliothek und die Definition des Standortes für die Universität sind sehr gute Beispiele für den ,weiten Blick', für eine im buchstäblichen Sinn umsichtige Planung: Sie schufen plötzlich eine klare, einfache Ausgangslage für die weitere Entwicklung der Bibliothek (die ja damals bereits seit 26 Jahren mit zahlreichen Studien, aber keinen Resultaten verfolgt worden war). Zur umsichtigen Planung gehörte, dass die Bedürfnisse und Wünsche der Bibliothek intensiv aufgenommen wurden, dass aber auch weitere Faktoren wie Stadt- und Kantonsbauplanung, Denkmalpflege, Hochschulentwicklung zu ihrem Recht kamen. Denn die Tatsache, dass dem Begleit- und Steuerungsgremium der zweiten Machbarkeitsstudie sowohl die politische Ebene von Stadt und Kanton als auch die Entscheidungsebene von Stadtplanung, kantonaler Baubehörde und Denkmalpflege angehörten, erleichterte die Entscheidungsfindung und vor allem die verbindliche Beschlussfassung enorm. Die Beschlüsse legten fest:

1. Die ZHB wird künftig auf zwei Hauptstandorte aufgeteilt sein, da sie am bestehenden Ort nicht erweitert werden kann und da der ,universitäre‘ Bibliotheksteil im Gebäude der Uni und der PH Luzern untergebracht werden kann. Oder mit anderen Worten: Die Erweiterung der ZHB wird an einem zweiten Standort, im Gebäude der Universität, realisiert.

2. Die Magazinbestände können mit keiner Lösung in einem Gebäude der Bibliothek in der Stadt behalten werden. Neben den Bibliotheksgebäuden für das allgemeine und für das universitäre Publikum mit grosszügigen Freihandbeständen muss ein Magazingebäude erbaut werden, das aber auch ausserhalb der Stadt stehen kann.

3. Das bestehende Hauptgebäude der Bibliothek im Park wird renoviert und für eine extensive Freihandnutzung und eine deutlich verbesserte Präsentation und Benutzung der Sondersammlung umgebaut. Es behält das gleiche Volumen wie bisher.

Was bedeutet das nun im Detail?

\section{Erweiterung}

Die Erweiterung der ZHB wurde im Gebäude der Universität und der PH Luzern realisiert. Sie umfasst beinahe ein ganzes Stockwerk, knapp 5’000 m², und sie bietet ca. 300’000 Bände in Freihandaufstellung und über 650 Arbeitsplätze für Benutzende sowie Arbeitsplätze für 34 Mitarbeitende der Bibliothek an. 


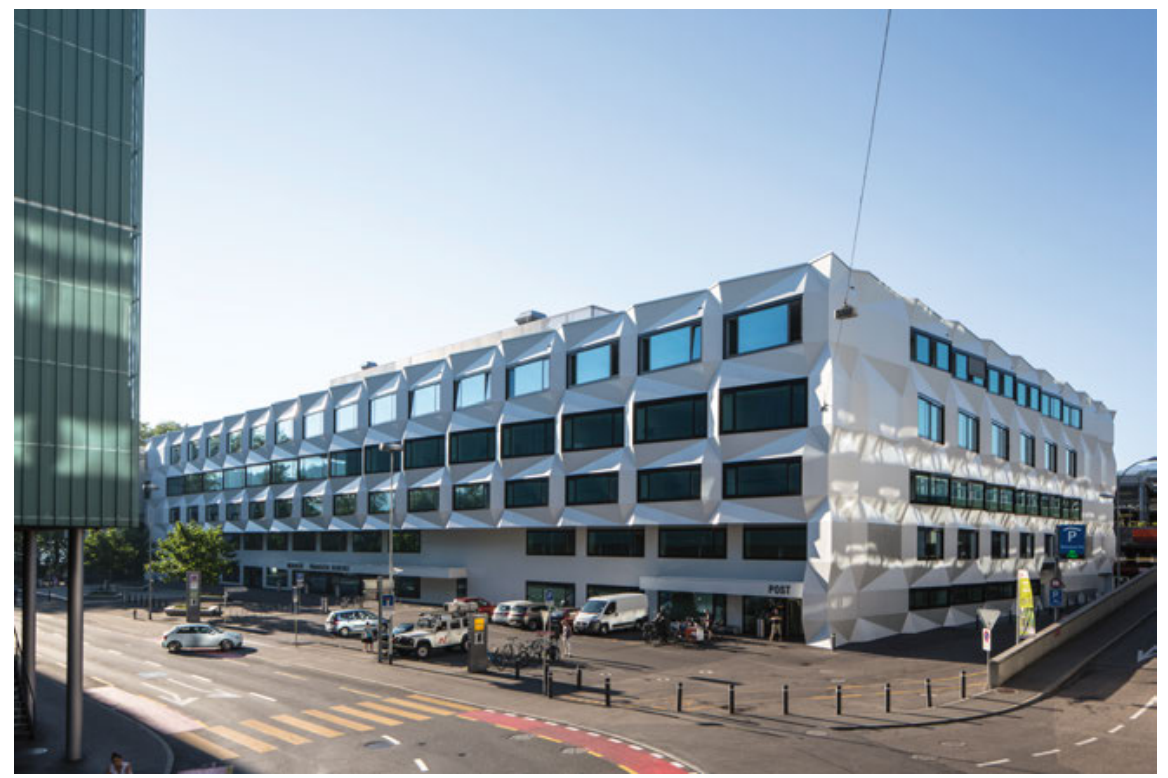

Abb. 4: Gebäude der Universität Luzern und der PH Luzern; die Bibliothek ist im 1. Geschoss, über der Post (C) Ulrich Niederer, ZHB Luzern)

Die Bibliothek liegt im 1. Geschoss, zwischen den Geschossen mit den grossen Hörsälen und der Mensa einerseits und den Geschossen mit den kleineren Seminarräumen und den Büros der PH Luzern und der Universität (2. bis 4. Geschoss) andererseits. Die ZHB war glücklicherweise von Anfang bis zum Schluss in die Planungsarbeiten mit einbezogen. Insbesondere aber konnte mit den Leitungen der beiden Institutionen früh eine einheitliche Haltung zur grundsätzlichen Organisation der Bibliothek erarbeitet werden, an der alle Beteiligten in allen Planungsschritten festhielten: vor allem ein Zugang in die Bibliothek für alle Benutzenden (auch für die Dozierenden!), eine Info- und Ausleihtheke, Mengengerüste für die Bestände der einzelnen Fächer sowie eine einheitliche Benutzungsordnung - Prinzipien, die wenig spektakulär scheinen, die aber manche Gespräche und einiges an Vertrauensbildung brauchten. Dieses Gebäude, und damit die Bibliothek, konnte fristgerecht fertiggestellt werden; es wurde im Sommer 2011 bezogen und am 01. September 2011 eröffnet. 


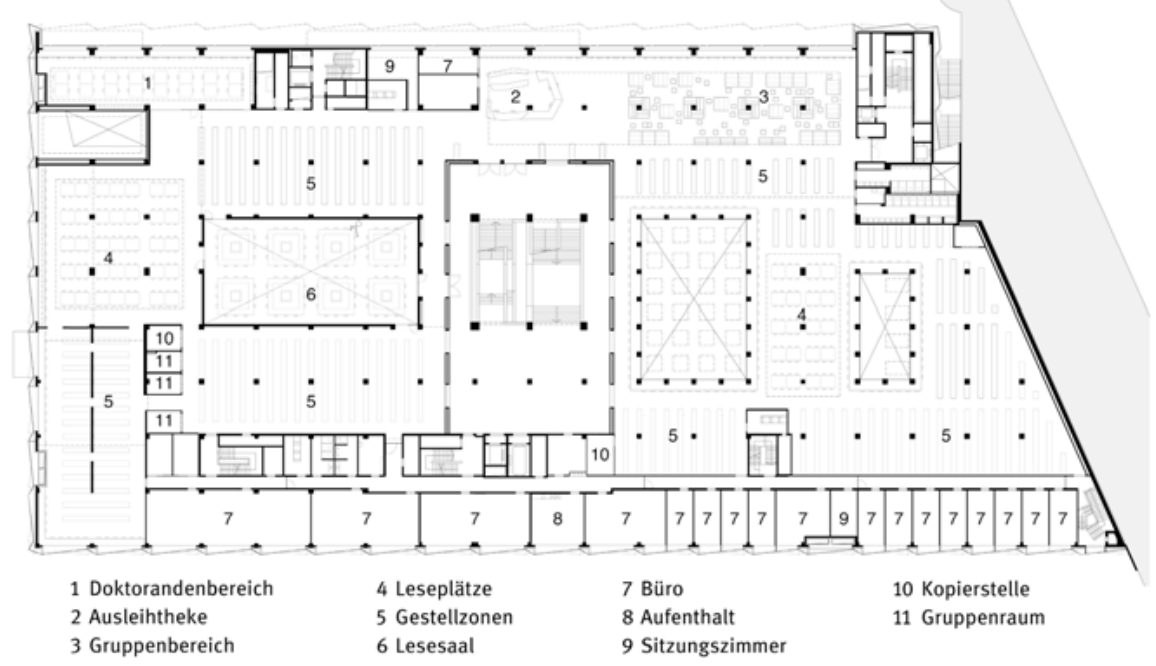

Abb. 5: Grundriss der Bibliothek im Uni-/PH-Gebäude, 1. OG (৫) Enzmann Fischer Architekten, Zürich)

\section{Vom Aussenlager zur Kooperativen Speicherbibliothek}

Für das Aussenlager führte eine Arbeitsgruppe unter der Leitung des kantonalen Hochbauamtes zwischen 2005 und 2007 detaillierte Untersuchungen verschiedener Varianten von Magazinierung durch mit dem Ziel, die beste langfristig gültige Archivierungsmöglichkeit in konservatorischer und ökonomischer Hinsicht zu finden. Sie kam zu dem Schluss, dass ein automatisiertes Behälterlager die optimale Variante darstellt; Vorbilder sind etwa das Magazin der norwegischen Nationalbibliothek in Mo i Rana, das seit 1998 in Betrieb und 2016 mit einem neuen Modul ergänzt wurde, oder vor allem das neue automatisierte Lager, das Additional Storage Building der British Library in Boston Spa, das 2007 eröffnet wurde und seither mit einem zweiten Modul erweitert wurde; ein drittes ist in Planung. 


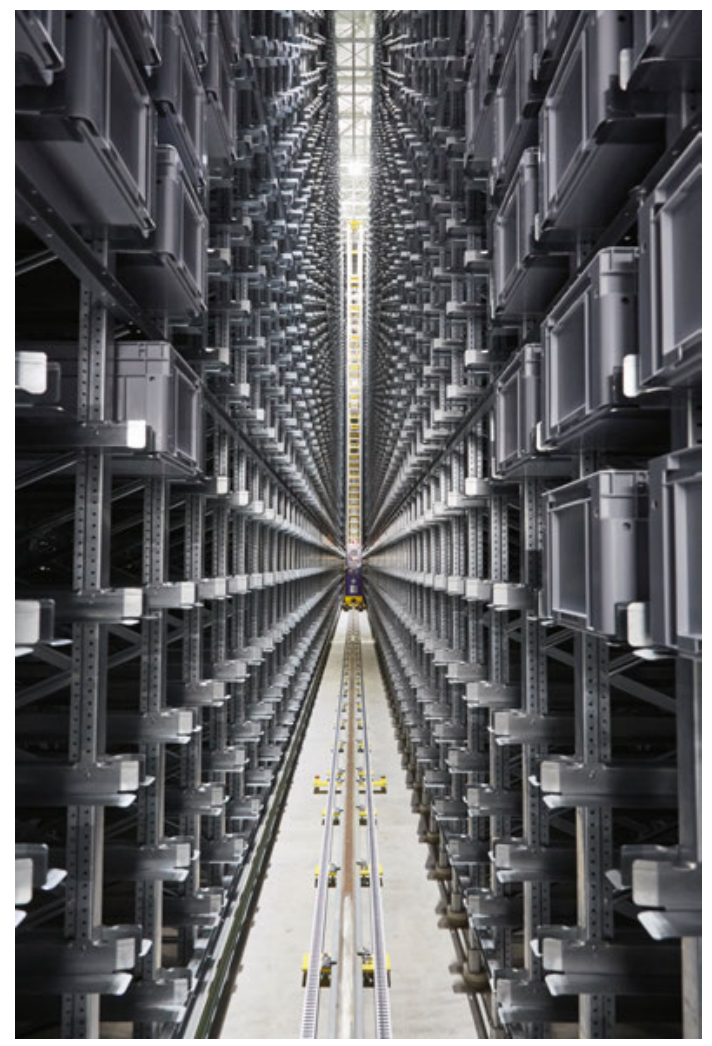

Abb. 6.1: Kooperative Speicherbibliothek Schweiz, eine Gasse im Lager der 14 Meter hohen Regale (@ Ulrich Niederer, ZHB Luzern)

Die Varianten im Vergleich waren ein traditionelles Lager mit festen respektive mit beweglichen Gestellen, ein Hochregallager des in den USA oft benutzten Typs ,Harvard Depository', wie es auch im Pariser Centre Technique du Livre de l'Enseignement Supérieur (CTLes) erstellt wurde, schliesslich ein vollständiges Outsourcing an einen externen, privatwirtschaftlichen Partner des Logistikbereichs.

Die Untersuchungen ergaben vor allem, dass ein automatisiertes Behälterlager ein bedeutendes zusätzliches Potenzial an ökonomischer und betrieblicher Verbesserung aufweist, wenn verschiedene Bibliotheken gemeinsam ein solches Lager bauen und betreiben. Deshalb beschlossen die Trägerschaften von schliesslich fünf Bibliotheken (Öffentliche Bibliothek der Universität Basel, ZHB Luzern, ZB Solothurn, Bibliotheken der Universität Zürich und die ZB Zürich), die Zusammenarbeit zu realisieren. Sie nahmen die Arbeit 2009 auf, die verbindlichen Zustimmungen lagen im Sommer 2014 vor. Mit der Bauausführung konnte ein 
Jahr später begonnen werden, im Februar 2016 nahm die Kooperative Speicherbibliothek den Betrieb auf und im Juni 2016 fand die offizielle Eröffnung statt.

Das ist eine sehr abgekürzte Darstellung. Ausführlicher, sowohl hinsichtlich der aufwendigen politischen Prozesse als auch der durchaus nicht trivialen bibliothekarischen Vorbereitungen, kann die Entstehungsgeschichte in anderen Publikationen $^{2}$ nachgelesen werden; hier bleibt festzuhalten, dass am Beginn des Projekts umsichtige Planung stand, dass aber die Kooperation, für die der weite Blick aller Beteiligten Voraussetzung war, dem Projekt den grossen Schub zur erfolgreichen Realisierung gab.

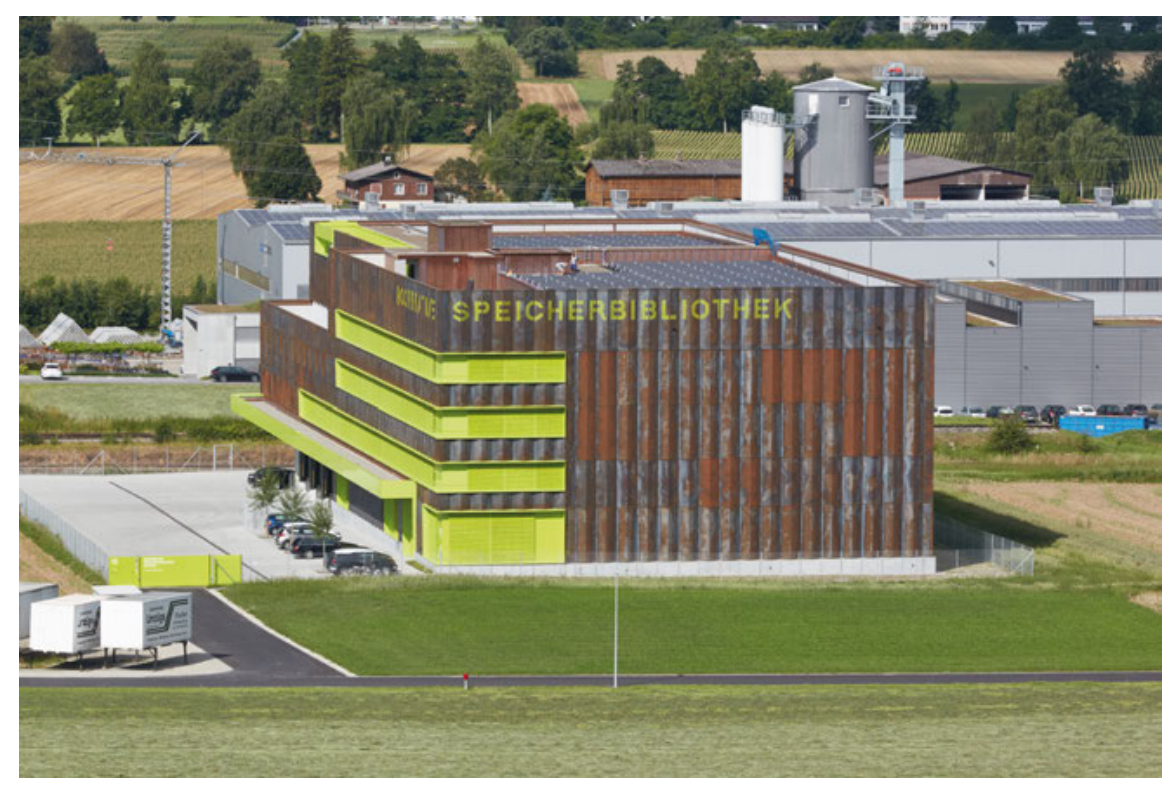

Abb. 6.2: Kooperative Speicherbibliothek Schweiz, Juli 2016 (C Ulrich Niederer, ZHB Luzern)

2 Ulrich Niederer: Hochregallager - ein neuer Weg der Magazinierung: die Kooperative Speicherbibliothek Schweiz. Eine Luzerner Fallgeschichte mit Exkursen. In: Petra Hauke, Klaus Ulrich Werner (Hrsg.): Praxishandbuch Bibliotheksbau: Planung - Gestaltung - Betrieb. Berlin/Boston 2016, S. 345-359 sowie Ulrich Niederer, Dani Tschirren: Die Kooperative Speicherbibliothek Schweiz - Erfahrungen nach einem Jahr Betrieb. In: ABI Technik 37/2 (2017), S. 103-110 


\section{Sanierung und Umnutzung des Hauptgebäudes ZHB}

Zur Sanierung und Umnutzung wurde 2007 ein Architekturwettbewerb durchgeführt. Das Siegerprojekt des Wettbewerbs mit internationaler Beteiligung stammt vom Generalplanerteam Caretta+Weidmann, Zürich, dem als Architekten das Büro Lussi+Halter, Luzern, angehört. Es sieht vor, den ganzen Magazintrakt zu einem Publikumsbereich umzugestalten; dazu wird dieser komplett ausgehöhlt und neu aufgebaut. Ein sehr geschickter Vorschlag der Architekten ist es, die Böden der Stockwerke nicht von Fassade zu Fassade zu ziehen, sondern an der Ostseite - zum ruhigen Innenhof hin - offen zu lassen. Die entstehende Galerie wird für Arbeitsplätze genutzt. Diese Öffnung bringt Licht und auch eine gewisse Leichtigkeit in diesen Gebäudeteil, der seinem Charakter als Magazin treu bleibt und deshalb geringe Raumhöhen (2,20 m) aufweist.

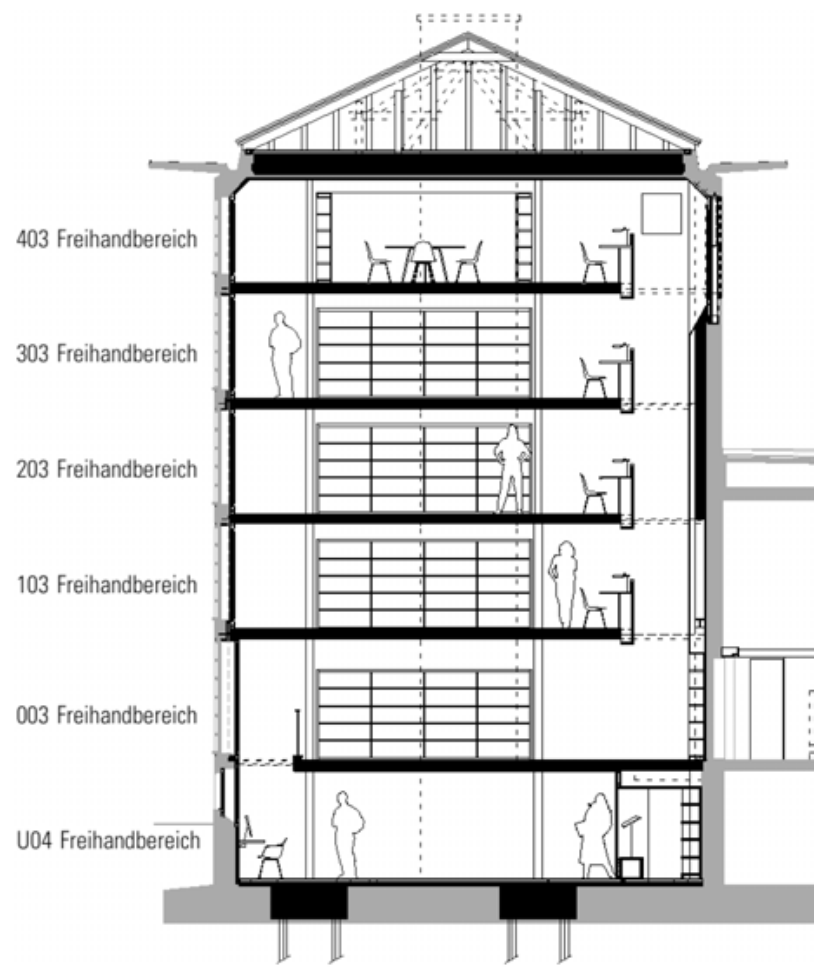

Abb. 7: Schnitt durch den neuen Freihandbereich im ehemaligen Magazintrakt (๔ ARGE Lussi+Halter, Luzern) 
Ein besonderes Highlight wird im Untergeschoss das grosse ,Schaufenster' sein, eine klimatisierte Vitrine von etwa $50 \mathrm{~m}^{2}$. Praktisch über die ganze Länge des Geschosses gezogen, wird sie für die Sondersammlung eine besonders attraktive Ausstellungsmöglichkeit schaffen.

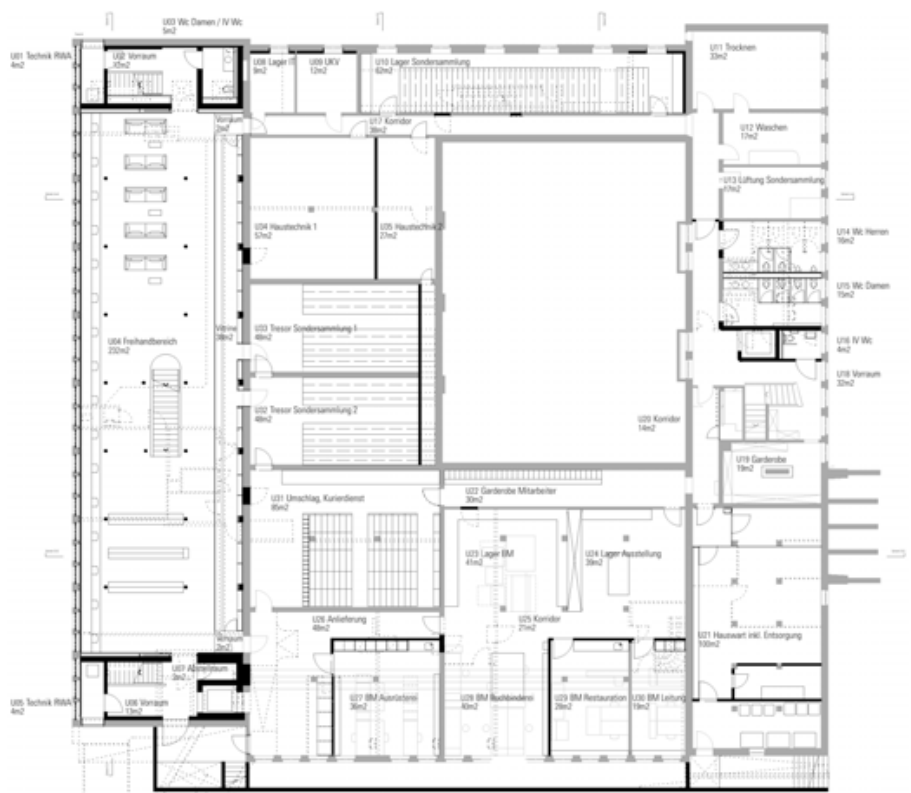

Zentral- und Hochschulbidioches Lwern Untergeschoss

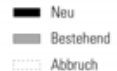

Abb. 8: Grundriss des Untergeschosses mit dem Schaufenster der Sondersammlung (৫) ARGE Lussi+Halter, Luzern)

Im Erdgeschoss wird neu eine Cafeteria für das Publikum und die Mitarbeitenden eingerichtet, der neue, grössere Lesesaal für die Sondersammlung untergebracht und ein besserer Platz für allgemeine Ausstellungen geschaffen. Im Übrigen wird der Verwaltungstrakt sanft renoviert; hier gibt es keine grösseren Umbauten. Er wird knapp 90’000 Bände in Freihandaufstellung und die Spezialmagazine für die Sondersammlung enthalten. Für Benutzende stehen 157 Arbeitsplätze zur Verfügung, davon $54 \mathrm{im}$ Lesesaal und $79 \mathrm{im}$ Freihandbereich sowie zusätzlich 4 Gruppenräume mit 24 Arbeitsplätzen, schliesslich 65 Büroarbeitsplätze für Mitarbeitende. 


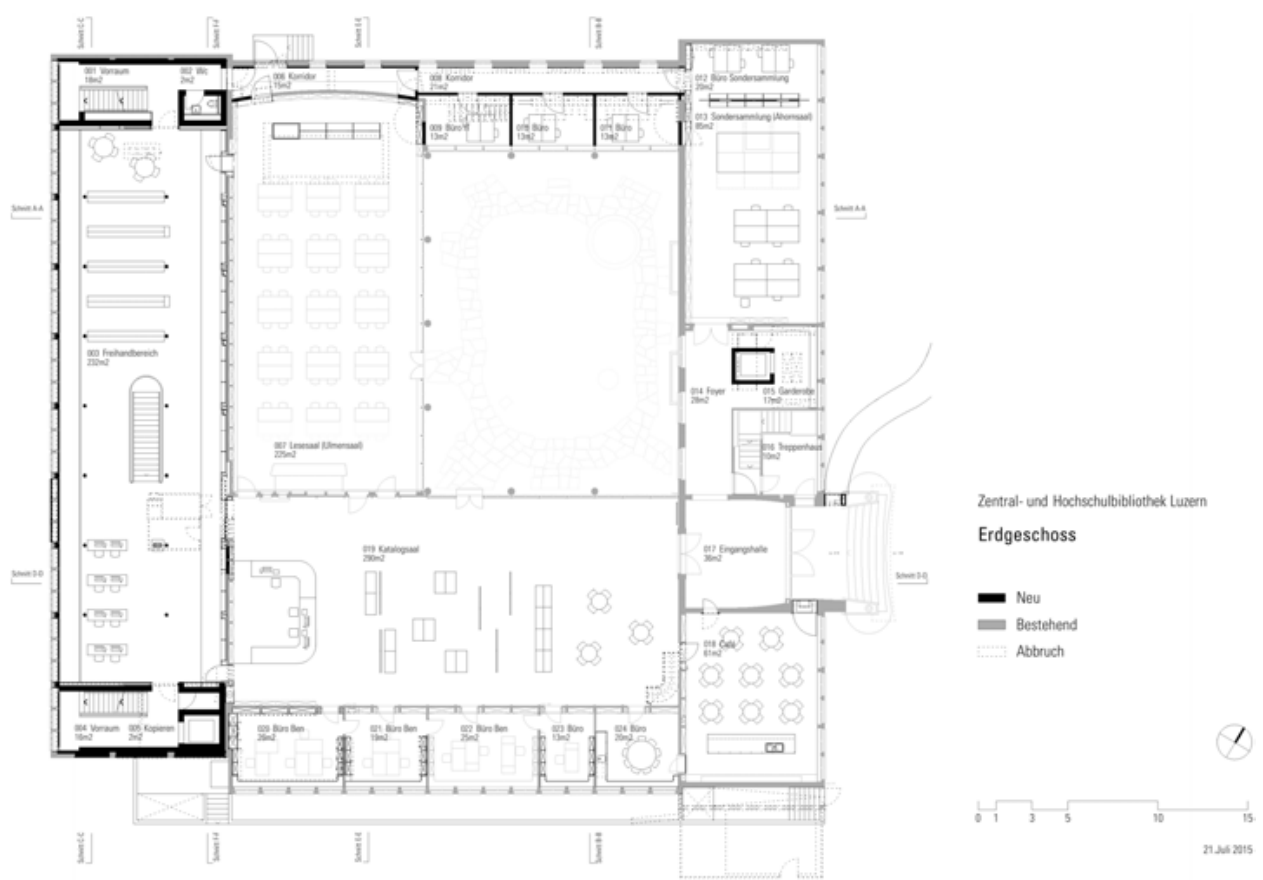

Abb. 9: Grundriss neues Erdgeschoss ZHB (৫ ARGE Lussi+Halter, Luzern)

Die bauliche Realisierung wird im Herbst 2011 beginnen und das neue Gebäude wird im Sommer 2013 eröffnet werden: So war die Planung in der Botschaft der Regierung an das Kantonsparlament angelegt, das dem Projekt im Juni 2010 mit einer Zweidrittelmehrheit zustimmte. Wir stiessen ein erstes Mal an, um die Realisierung des letzten Teils des Plans zur Entwicklung der ZHB und damit der sicheren, zeitgemässen bibliothekarischen Versorgung der Luzerner Hochschullandschaft und des allgemeinen Publikums zu feiern. Der weite Blick hatte sich auf allen Ebenen behaupten können...

Was dann folgte, ist ein Lehrstück zum Primat der Politik. Im Winter 2010 beschied das Parlament der Regierung, es seien aus finanzpolitischen Gründen nicht genügend finanzielle Mittel verfügbar, um alle geplanten Bauvorhaben zu realisieren. Die Regierung musste daraufhin die Ausführung eines der grossen Projekte um zwei Jahre verschieben; die Wahl fiel auf das Projekt zum Hauptgebäude der ZHB. Im Winter 2011, als das Projekt zur Freigabe des Baubeginns Ende 2012 wieder dem Parlament vorgelegt wurde, verweigerte das Parlament der Regierung das Budget und nahm zudem den Antrag eines Parlamentariers an, der vorschlug, anstelle des bestehenden Gebäudes der ZHB einen grösseren Neubau im Public Private Partnership-Verfahren (PPP) zu errichten; dem privaten Partner 
solle die Nutzung des grossen Teils des Gebäudes zustehen, er würde dafür die Fläche für die Bibliothek dem Kanton kostenfrei zur Verfügung stellen. In der aufgeladenen Atmosphäre der Budgetdiskussion im Parlament fand dieser Vorschlag, mit dem dem Kanton die als überrissen bezeichneten Renovationskosten für das als nicht erhaltenswert bezeichnete Gebäude erspart werden könnten, eine deutliche Mehrheit. Im Lauf des folgenden Jahres wurden das Projekt und vor allem die zugrundeliegenden Kostenberechnungen durchaus kontrovers und skeptisch diskutiert. Aber gegen Ende 2012 fand ein weiterer Parlamentsvorstoss Zustimmung: Der Kanton solle das Gebäude neu und grösser bauen und darin neben der Bibliothek auch die kantonalen Gerichte unterbringen. Damit war zwar der frühere Beschluss des PPP-Neubaus abgeschrieben - wohl zur Erleichterung manch eines ehemaligen Befürworters. Aber der Kanton stand gleichwohl in der Pflicht, einen Neubau zu planen, auch wenn inzwischen ein Antrag vorlag, das Gebäude unter Schutz zu stellen, verschiedene Fach- und alle Planerverbände sich strikt gegen einen Abriss aussprachen und zwischen Stadt und Kanton Luzern durchaus unterschiedliche Interpretationen der legalen Basis für einen Neubau zu klären waren. Als die Dienststelle Immobilien, das Hochbauamt des Kantons Luzern, den Wettbewerb für den Neubau ausschreiben wollte, musste sie feststellen, dass sie keine Jury zusammenstellen konnte - kein Architekt, kein Ingenieur fand sich, der dem Gremium angehören mochte! Zudem war inzwischen in der Stadt eine Initiative zustande gekommen, die den Erhalt des Gebäudes und damit die Erhaltung des umgebenden Stadtparks verankern wollte. Der Kanton verschob daher weitere Aktivitäten bis nach der städtischen Abstimmung über diese Initiative; sie fand im November 2014 statt und ergab eine Dreiviertelmehrheit für den Erhalt des Gebäudes.

Damit blieb wirklich nur noch der Weg der Renovierung; die Pläne wurden reaktiviert und sanft aktualisiert - es erwies sich, dass das Projekt nach Überzeugung auch der ZHB immer noch zu einer modernen, gut für die Zukunft gerüsteten Bibliothek führen würde - und Anfang 2016 dem Parlament neuerlich zur Bestätigung vorgelegt. Das Parlament stimmte zu. Die Ausführungs- und Umzugsplanung wurde konkret, als Baubeginn Ende Februar 2017 festgelegt, als erster Umzug die Sondersammlung in ein Provisorium im Staatsarchiv verschoben. Dann erhielt das Budget für 2017 erneut keine Zustimmung im Parlament, was unter anderem einen Ausführungsstopp von Bauprojekten, auch unserer ZHB, mit sich brachte. Der budgetlose Zustand dauert an; zum Zeitpunkt des Schreibens, Mitte August 2017, scheint es, als könnte Ende September ein genehmigtes Budget vorliegen, was die Ausführung auslöste. Könnte... 


\section{Fazit}

Erfolg braucht Geduld und ohne Hartnäckigkeit geht es sehr selten. Aber das sind allenfalls notwendige Eigenschaften, noch keine hinreichenden Faktoren - dazu gehören der weite Blick, die umsichtige Planung. Dieser kurze Bericht einer langen Geschichte zeichnet die Fährnisse, denen ein Projekt unterworfen sein kann, nach. Der Bericht, es muss gesagt sein, erzählt nichts von den emotionalen Achterbahnfahrten, denen insbesondere die Belegschaft und das Publikum der Bibliothek durch die mehrfach wiederholten Stillstellungen des dritten Teilprojekts in den letzten zwölf Jahren ausgesetzt war; das wäre eine eigene und nicht ganz einfache Geschichte.

Im Hinblick auf die Planung aber zeigt das Gesamtprojekt beinahe lehrbuchhaft, wie sie verläuft und welche Bedingungen und Einflussfaktoren zum Erfolg führen können, welche Rolle die fachliche Planung spielen kann und welche die Politik, d.h. die Entscheidungsebene der Exekutive, das Parlament als Legislative, allenfalls auch der Souverän, wenn Volksabstimmungen notwendig werden. Und das Projekt zeigt auch, dass der weite Blick zwar Erfolg begründen, aber auch an Grenzen stossen kann. Alle drei dargestellten Teilprojekte beruhen auf dem gleichen Rahmenkonzept, dessen Umsicht erst die erfolgreiche Realisierung ermöglichte; zwei konnten innerhalb kurzer Frist zum Erfolg geführt werden, das dritte mit der längsten Dauer stiess mehrfach auf Schwierigkeiten, Sandbänke, Untiefen, die mit dem schlicht gegebenen Primat der Politik zusammenhängen. Es ist noch immer nicht beendet, unbekümmert um die hohen Kosten, die die Verschiebungen jeweils mit sich brachten.

Der weite Blick wird deshalb, gerade bei den komplexen Entwicklungen, die sich heute im schweizerischen Bibliothekswesen abzeichnen, an Gewicht zunehmen: Die Notwendigkeit, umsichtige Planung mit all ihren Faktoren deutlich zu machen, gehört stärker denn je zu den Hauptaufgaben der Bibliotheksleitungen. Das vorgelebt und mit Nachdruck verfolgt $\mathrm{zu}$ haben, gerade im Rahmen der grossen nationalen Projekte, ist ein lebendiges Verdienst von Susanna Bliggenstorfer und dafür ist ihr das ganze schweizerische Bibliothekswesen, sind wir alle einfach sehr dankbar. 